\title{
Analysis of the solar cycle and core rotation using 15 years of Mark-I observations: 1984-1999
}

\section{The solar cycle}

\author{
S. J. Jiménez-Reyes ${ }^{1,2}$, T. Corbard ${ }^{1, \star}$, P. L. Pallé ${ }^{2}$, T. Roca Cortés ${ }^{2,3}$, and S. Tomczyk ${ }^{1}$ \\ 1 High Altitude Observatory, NCAR, PO Box 3000, Boulder, CO 80307, USA \\ 2 Instituto de Astrofísica de Canarias, 38701, La Laguna, Tenerife, Spain \\ 3 Departamento de Astrofísica, Universidad de La Laguna, Tenerife, Spain
}

Received 3 April 2001 / Accepted 2 October 2001

\begin{abstract}
High quality observations of the low-degree acoustic modes ( $p$-modes) exist for almost two complete solar cycles using the solar spectrophotometer Mark-I, located at the Observatorio del Teide (Tenerife, Spain) and operating now as part of the Birmingham Solar Oscillations Network (BiSON). We have performed a Fourier analysis of 30 calibrated time-series of one year duration covering a total period of 15 years between 1984 and 1999. Applying different techniques to the resulting power spectra, we study the signature of the solar activity changes on the low-degree $p$-modes. We show that the variation of the central frequencies and the total velocity power $(T V P)$ changes. A new method of simultaneous fit is developed and a special effort has been made to study the frequency-dependence of the frequency shift. The results confirm a variation of the central frequencies of acoustic modes of about $0.45 \mu \mathrm{Hz}$, peak-to-peak, on average for low degree modes between 2.5 and $3.7 \mathrm{mHz}$. The TVP is anti-correlated with the common activity indices with a decrease of about $20 \%$ between the minimum and the maximum of solar cycle 22. The results are compared with those obtained for intermediate degrees, using the LOWL data. The frequency shift is found to increase with the degree with a weak $\ell$-dependence similar to that of the inverse mode mass. This verifies earlier suggestions that near surface effects are predominant.
\end{abstract}

Key words. Sun: activity - Sun: oscillations - Sun: interior - Sun: rotation - methods: data analysis

\section{Introduction}

Understanding the observed solar variability is one of the major goals of solar physics. Because the frequency shifts of solar $p$-modes are known to be very sensitive to the solar activity cycle, the analysis of helioseismic data has been used to track those physical processes which underly the origin of the cyclic changes observed at the solar surface. Helioseismology based on low-degree $p$-modes is necessary to look for potential structure or dynamic changes in the deep interior.

The first report of frequency shifts of the low-degree $p$-modes was given by Woodard \& Noyes (1985). Using ACRIM data, they found that the few observed $\ell=0$ and 1 modes presented a change in the central frequency of $0.42 \pm 0.14 \mu \mathrm{Hz}$ in average during the declining phase of cycle 21 (1980-1984). These results were confirmed by

\footnotetext{
Send offprint requests to: S. J. Jiménez-Reyes, e-mail: sjimenez@ll.iac.es

* Present address: Institute of Astronomy, University of Cambridge, Madingley Road, Cambridge CB3 OHA, UK.
}

Fossat et al. (1987) by comparison with observations made at the south pole, and by Pallé et al. (1989) using a long set of data from the Mark-I instrument at Observatorio del Teide covering the full cycle 21 (1977-1988).

Subsequently, Régulo et al. (1994) used Doppler observations collected from the maximum of cycle 21 to the falling phase of cycle 22 (1980-1993), obtained with Mark-I instrument, to calculate monthly frequency shifts. They showed that, for all low degree acoustic modes, there is an important frequency shift of $0.52 \pm 0.02 \mu \mathrm{Hz}$ correlated with solar activity. In addition, the amplitude of these variations is different when $\ell=1,3$ and $\ell=0,2$ are considered separately. The odd modes present, on average, a change of $0.58 \pm 0.06 \mu \mathrm{Hz}$; the even ones show a full shift of only $0.33 \pm 0.06 \mu \mathrm{Hz}$.

Two other important properties of the low-degree changes have also been pointed out recently. Anguera Gubau et al. (1992) observed the frequency dependence of the frequency shifts for the low-degree $p$-modes, (later confirmed by Chaplin et al. 1998) in agreement with the earlier results of Woodard et al. (1991) for 
intermediate degrees. Jiménez-Reyes et al. (1998) found that the frequency shifts, when plotted against an activity index, show a hysteresis behavior rather than a simple linear correlation. This result was interpreted as part of structural changes associated with the solar activity which are taking place in the Sun. This must be confirmed by more observations but the interpretation of these later results as being partly due to structural changes in the interior associated with the solar activity has been found to be a complex problem. Recently, Moreno-Insertis \& Solanki (2000) have studied in detail the signature left on the lowdegree $p$-mode frequencies by the surface solar magnetic activity. Whether these changes are taking place only close to the surface or not is not completely clear and one of the requirements to address this question is to get precise and reliable measures of the low degree mode parameters for a long period of time.

The spectrophotomer Mark-I, has been collecting solar observations for almost two complete solar cycles. The available database for low-degree $p$-modes, probably the longest in duration and the most stable, is used in the present work to analyze the signature of the solar cycle in the mode parameters and to parameterize the observed frequency shifts as a function of various classical solar indices.

In the following section, the essential steps of the data reduction leading to the yearly spectra are presented. In general, the frequency shift between a time $t_{i}$ and a time $t_{\mathrm{o}}$ taken as reference, can be written as a function of the frequency and the degree, i.e. $\delta \nu\left(t-t_{\mathrm{o}}, \nu, \ell\right)$. In the following the reference time is 1986 which corresponds to a minimum of solar activity. In Sect. 3, the integrated frequency shift $\Delta \nu^{i} \equiv<\delta \nu\left(t_{i}-t_{\mathrm{o}}, \nu, \ell\right)>$ is analyzed where the brackett indicates an average for all observed low degrees and frequencies between 2.5 and $3.7 \mathrm{mHz}$. Two techniques are proposed to measure the frequency shift from the cross-correlation function between power spectra of time-series created at different solar activity level. In addition to the frequency shift, the second technique allows us to study the time variation of the total velocity power $(T V P)$ which are presented in Sect. 4. Then, in Sect. 5, we focus on the study of the frequency dependence of the frequency shifts. Again, two different techniques are used. The first one consist in simply cutting the spectra in band of $135 \mu \mathrm{Hz}$ before computing the cross-correlation functions. The second one is a new procedure developed here and called simultaneous fitting: all the yearly spectrum are fitted at the same time assuming that the time dependence of the mode frequencies can be described as a linear function of the radio flux at $10.7 \mathrm{~cm} F_{10}^{i}$, taken as solar activity index i.e.:

$\delta \nu\left(t_{i}-t_{\mathrm{o}}, \nu, \ell\right)=\delta \nu(\nu, \ell)\left(F_{10}^{i}-F_{10}^{\mathrm{o}}\right)$,

where $F_{10}^{\mathrm{o}}$ represents the radio flux at the 1986 solar minimum. In addition, we define $\delta \nu(\nu)$ as the frequency shift per radio flux unit averaged over $\ell$. We note that if we had used shorter time-series and a magnetic index instead of the radio flux to parameterize the time dependence of the frequency shift, a more complicated formulation would probably have been needed in order to take into account the hysteresis behaviour found when magnetic indices are plotted versus the frequency shift during the cycle (Jiménez-Reyes et al. 1998).

In order to check the $\ell$-dependence of the frequency shift, we use the fact that pairs of low-degree $p$-modes with the same parity have "almost" the same frequency and, that they are equally spaced in frequency. This allows us to provide in Sects. 3, 4, 5 not only the $\ell$-averaged quantities defined above $(\Delta \nu, T V P, \delta \nu(\nu))$ but also the quantities related to even and odd modes separately i.e. respectively: $\Delta \nu_{0,2}, T V P_{0,2}, \delta \nu(\nu)_{0,2}$ and $\Delta \nu_{1,3}, T V P_{1,3}$, $\delta \nu(\nu)_{1,3}$. Finally, in Sect. 6 the $\ell$-dependence analysis of the frequency shift is completed by comparing the results with those obtained at higher degrees $(\ell=1,99)$ using the LOWL database.

\section{Observations and data analysis}

The data used in this work come from the observations carried out at the Observatorio del Teide between 1984 and 1999. The observations consist of daily measurements of the solar radial velocity obtained with the Mark-I resonant scattering spectrophotometer. This instrument has been sited at the Observatorio del Teide since 1975. After some hardware updates in 1984, the experiment has been running without interruption other than bad weather and instrumental failures. The data reduction process is explained in more detail elsewhere (van der Raay et al. 1985; Pallé et al. 1986, 1993). Briefly, the data are corrected from the annual scan (Earth's orbit around the Sun) of the nonlinear solar line shape, and calibrated by fitting the known daily velocity of the observatory; only two parameters are fitted, taken to be the same over the all 15 years analyzed here. Then, the daily residuals are joined in consecutive 360 days leading to a total of 30 time-series with 6 months in common between consecutive series. Although all series are not independent, they show very similar duty cycles (around 25\%). Finally, the corresponding power spectra were calculated for every time-series using a traditional Fourier analysis. All the spectra show the peaks of the lowdegree $p$-modes with $\ell \leq 3$. The typical sideband structure appears in the spectra at $k_{D}=11.57 \mu \mathrm{Hz}$ as a direct consequence of the observing window achieved from just one station.

\section{Frequency integrated frequency shifts}

Once the power spectrum of each time-series has been calculated, the integrated frequency shifts $\Delta \nu^{i}$ are determined. This is done by computing the cross-correlation $\rho^{i}\left(\nu_{j}\right)$ of each power spectrum $i$ with the power spectrum of the time-series covering 1986 taken as reference. As we show in Sect. 5, the dependence of the frequency shift for low and intermediate-degree $p$-modes appears to be essentially null below $2 \mathrm{mHz}$, whereas at high frequency (above $3.7 \mathrm{mHz}$ ) the frequency shift is expected to drop 
quickly. The analysis of the integrated changes is limited to the modes between 2.5 and $3.7 \mathrm{mHz}$. Considering a larger interval could bias the results by including the strong high frequency variations that are less accurately determined and have possibly different physical explanation (Goldreich et al. 1991).

In order to calculate the position of the crosscorrelation main peak, two different methods have been used. The first was used in previous analyses (Régulo et al. 1994; Jiménez-Reyes et al. 1998) and takes the maximum of a second-order polynomial fitted to the logarithm of the cross-correlation function in an interval $\pm \sigma$ around the main peak (where $\sigma$ is the second-order moment). This cross-correlation is calculated starting at an appropriate lag for which the function is symmetric, and which is obtained by calculating the third-order moment. This procedure directly provides a value of the mean frequency shift between the $\ell \leq 3 p$-modes and the corresponding values at solar activity minimum, the chosen reference.

The second method, introduced here, is based on the shape of the cross-correlation function. Assuming that each oscillation mode can be modeled by a damped harmonic oscillator, each peak in the spectra has a Lorentzian profile and the correlation function has also a Lorentzian profile. In order to improve the determination of the frequency shift, the model also take into account the presence of sidebands with amplitude $\beta_{|1|}$ located at $\pm 11.57 \mu \mathrm{Hz}$. Thus, our model of the cross-correlation function between the spectrum $i$ and the reference spectrum can be written as:

$M_{\rho}^{i}\left(\nu_{j}\right)=\sum_{k=-1}^{1} \beta_{|k|} \frac{A^{i}\left(\Gamma^{i} / 2\right)^{2}}{\left(\nu_{j}-\Delta \nu^{i}+k_{D} \cdot k\right)^{2}+\left(\Gamma^{i} / 2\right)^{2}}+B^{i}$

where the parameters to be fitted are:

- $A^{i}$, the amplitude of the central peak;

- $\Gamma^{i}$, the linewidth of the Lorentzian profile;

- $\Delta \nu^{i}$, the average frequency shift in the chosen frequency interval;

- $B^{i}$, the constant background level;

$-\beta_{|k|}$, the ratio of the sidebands to the central peak $(\beta=1$ for $k=0)$;

- $k_{D}=11.57 \mu \mathrm{Hz}$ is the constant separation of the sidebands and it is the only fixed parameter in the fit.

The best estimation, in the least square sense, of the parameters related to each time-series $i$, is obtained by minimizing the following quantities:

$\chi_{i}^{2}=\sum_{j=1}^{N}\left|\rho^{i}\left(\nu_{j}\right)-M_{\rho}^{i}\left(\nu_{j}\right)\right|^{2}$,

where $N$ is the total number of frequency bins in an interval of $\pm 20 \mu \mathrm{Hz}$ around the main peak of the crosscorrelation function. We applied a Levenberg-Marquard method (Press et al. 1992) but any other minimization routine may be used.

We note that the first technique is more objective in the sense that it does not require a physical hypothesis or

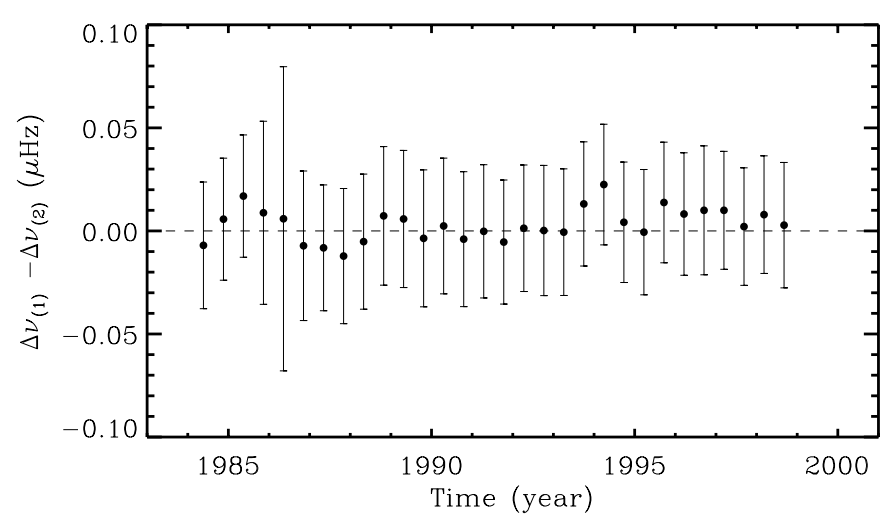

Fig. 1. Differences between the integrated frequency shifts as inferred from the cross-correlation functions by the two methods explained in the text i.e. (1) fit by a polynomial close to the maximum and (2) fit by a Lorentzian profile including side bands. The differences remain small and not significant.

modeling of the $p$-mode excitation and damping mechanisms. It uses only information contained in the spectrum while the second method assumes symmetric Lorentzian profiles. There is some evidence that the shape of the peaks in the power spectra are slightly asymmetric (Thiery et al. 2000). In order to check the efficiency of both methods and the influence of the duty cycle in the final result, we analyzed the frequency shifts in periods of 36 days for which the duty cycle varies. The differences between the two methods remain in general within the error bars. The only significant differences are found for time-series with very small duty cycle (around 10\%) but, for the yearly time-series analysed here, the duty cycle is moderately high and quite stable (around 25\%) from year to year. Regarding to the observed asymmetry of the $p$-modes, they are not thought to be important for this analysis. The second method takes into account the known distance from the sidebands to the main peak $( \pm 11.57 \mu \mathrm{Hz})$ which improve the results. Moreover this method allows to study not only the frequency shift but also the TVP by providing amplitudes and widths of the cross-correlation functions (see Sect. 4). Therefore hereafter only the second method will be considered but, for completeness, Fig. 1 shows the marginal differences between the frequency shifts obtained by applying both methods to the yearly time-series.

As mentioned in the introduction, the asymptotic theory predicts that, for low-degree $p$-modes, pairs of modes with alternately odd and even degrees are equally spaced in frequency with a separation of about $67 \mu \mathrm{Hz}$ i.e. half of the so-called big separation (e.g. Deubner \& Gough 1984). The contributions of even $(\ell=0,2)$ and odd degrees $(\ell=1,3)$ to the integrated frequency shift can therefore be separated simply by applying a mask to the spectra before using the procedure explained above. It should also be noted that, for full disk observations, the contribution of the two modes of a pair is not the same, due to the geometry of the modes at the surface. In the case of the odd modes, the frequency shifts come essentially from $\ell=1$ due to the high ratio in sensitivity between $\ell=3$ 
Table 1. Correlation coefficients between different solar indices and the yearly frequency shift. $r_{\mathrm{P}}$ is the Pearson linear correlation coefficient, $r_{\mathrm{S}}$ the Spearman rank correlation coefficient and $P_{\mathrm{s}}$ is the probability of having no correlation.

\begin{tabular}{|c|c|c|c|c|c|c|c|c|c|}
\hline \multirow[t]{2}{*}{ Index } & \multicolumn{3}{|c|}{$\Delta \nu$} & \multicolumn{3}{|c|}{$\Delta \nu_{0,2}$} & \multicolumn{3}{|c|}{$\Delta \nu_{1,3}$} \\
\hline & $r_{\mathrm{P}}$ & $r_{\mathrm{S}}$ & $P P_{\mathrm{s}}$ & $r_{\mathrm{P}}$ & $r_{\mathrm{S}}$ & $P_{\mathrm{s}}$ & $r_{\mathrm{P}}$ & $r_{\mathrm{S}}$ & $P_{\mathrm{s}}$ \\
\hline$R_{\mathrm{I}}$ & 0.94 & 0.88 & $9 \times 10^{-11}$ & 0.76 & 0.70 & $1 \times 10^{-5}$ & 0.90 & 0.85 & $4 \times 10^{-9}$ \\
\hline$F_{10}$ & 0.94 & 0.87 & $4 \times 10^{-10}$ & 0.77 & 0.72 & $7 \times 10^{-6}$ & 0.90 & 0.83 & $1 \times 10^{-8}$ \\
\hline KPMI & 0.90 & 0.86 & $8 \times 10^{-10}$ & 0.79 & 0.71 & $1 \times 10^{-5}$ & 0.85 & 0.84 & $8 \times 10^{-9}$ \\
\hline MPSI & 0.94 & 0.88 & $5 \times 10^{-10}$ & 0.83 & 0.76 & $2 \times 10^{-6}$ & 0.88 & 0.85 & $1 \times 10^{-8}$ \\
\hline TSI & 0.89 & 0.89 & $3 \times 10^{-8}$ & 0.78 & 0.78 & $1 \times 10^{-5}$ & 0.83 & 0.83 & $2 \times 10^{-7}$ \\
\hline $\mathrm{He}$ & 0.94 & 0.88 & $9 \times 10^{-11}$ & 0.79 & 0.74 & $2 \times 10^{-6}$ & 0.89 & 0.83 & $2 \times 10^{-8}$ \\
\hline
\end{tabular}
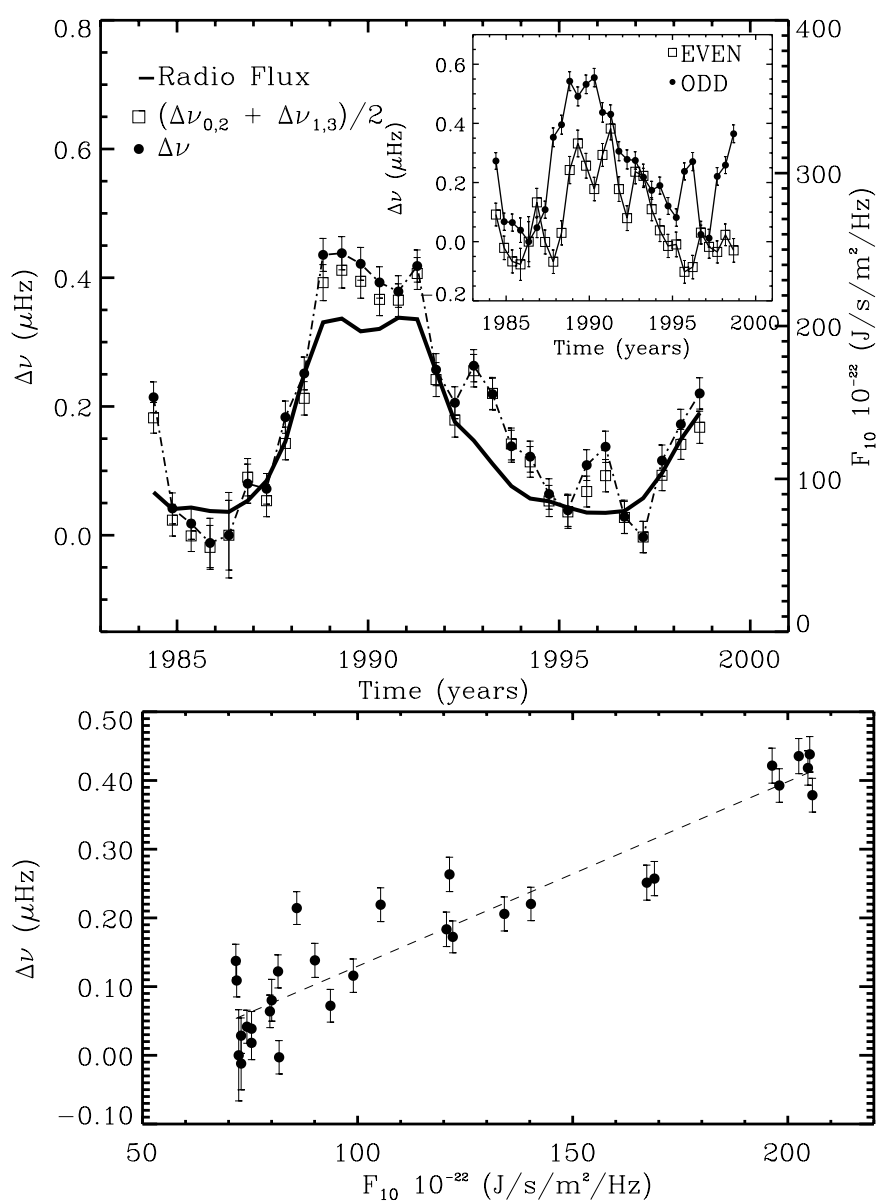

Fig. 2. Time variation of the integrated frequency shift for low-degree $p$-modes is plotted in the upper panel (black dots), where the radio flux at $10.7 \mathrm{~cm}$ is also shown (full line). In the sub plot, the results for the even and odd degrees separately are presented. The averages of these, are plotted in the main panel (squares). The lower figure shows the good linear correlation between the frequency shift and the radio flux.

and $1(\sim 0.1)$ whereas, in the case of the even modes the sensitivity ratio is close to one and therefore the contributions of $\ell=0$ and 2 are nearly the same.

Figure 2 illustrates the frequency averaged frequency shift between 2.5 and $3.7 \mathrm{mHz}$. The results corresponding to $\ell=0,2$ and $\ell=1,3$ have been plotted in the sub plot at the top-right corner and their average is shown in the main figure. The solid line represents the radio flux
Table 2. Intercept and slope of the frequency shift expressed as a linear function of different solar indices.

\begin{tabular}{llcc}
\hline $\begin{array}{l}\text { Solar } \\
\text { Index }\end{array}$ & $\begin{array}{c}\text { Intercept } a \\
(\mathrm{nHz})\end{array}$ & $\begin{array}{c}\text { Slope } b \\
\left(\mathrm{nHz} \text { per activity unit }{ }^{*}\right)\end{array}$ \\
\hline$R_{\mathrm{I}}$ & $\Delta \nu$ & $18.25 \pm 14.77$ & $2.56 \pm 0.18$ \\
& $\Delta \nu_{0,2}$ & $-48.08 \pm 25.94$ & $1.98 \pm 0.32$ \\
& $\Delta \nu_{1,3}$ & $60.62 \pm 21.51$ & $2.91 \pm 0.26$ \\
\hline$F_{10}$ & $\Delta \nu$ & $-139.07 \pm 24.17$ & $2.69 \pm 0.19$ \\
& $\Delta \nu_{0,2}$ & $-173.73 \pm 41.78$ & $2.11 \pm 0.32$ \\
& $\Delta \nu_{1,3}$ & $-115.88 \pm 36.40$ & $3.03 \pm 0.28$ \\
\hline KPMI & $\Delta \nu$ & $-98.43 \pm 27.42$ & $22.92 \pm 2.04$ \\
& $\Delta \nu_{0,2}$ & $-154.75 \pm 37.20$ & $19.08 \pm 2.78$ \\
& $\Delta \nu_{1,3}$ & $-63.15 \pm 40.42$ & $25.34 \pm 3.02$ \\
\hline MPSI & $\Delta \nu$ & $33.79 \pm 14.04$ & $149.06 \pm 10.46$ \\
& $\Delta \nu_{0,2}$ & $-39.06 \pm 21.10$ & $124.22 \pm 15.59$ \\
& $\Delta \nu_{1,3}$ & $79.81 \pm 22.19$ & $164.63 \pm 16.56$ \\
\hline TSI & $\Delta \nu$ & $-528.06 \pm 51.63$ & $386.71 \pm 37.80$ \\
& $\Delta \nu_{0,2}$ & $-437.13 \pm 65.43$ & $320.07 \pm 47.90$ \\
& $\Delta \nu_{1,3}$ & $-585.31 \pm 71.58$ & $428.67 \pm 52.40$ \\
\hline He & $\Delta \nu$ & $-438.86 \pm 42.45$ & $10.92 \pm 0.73$ \\
& $\Delta \nu_{0,2}$ & $-417.59 \pm 73.56$ & $8.87 \pm 1.26$ \\
& $\Delta \nu_{1,3}$ & $-447.34 \pm 67.87$ & $12.21 \pm 1.17$ \\
\hline \multirow{2}{*}{ Units: $\mathrm{nHz} ; \mathrm{nHz} /\left(10^{-22} \mathrm{~J} / \mathrm{s} / \mathrm{m}^{2} / \mathrm{Hz}\right), \mathrm{nHz} \mathrm{G}^{-1}, \mathrm{nHz} \mathrm{G}^{-1}}$, \\
$\mathrm{nHHz} \mathrm{W}^{-1} \mathrm{~m}^{2}, \mathrm{nHz} \AA^{-1}$ respectively.
\end{tabular}

averaged over the same periods as the time-series used for this work.

Although there are few departures from the general trend which do not agree with the smooth behavior of the solar index, the integrated signal concurs very well with the radio flux, which represents here the behavior of the solar cycle. The average of both contributions $(\ell=0,2$ and $\ell=1,3$ ) follows well the integrated signal as expected. The amplitude, measured as the straight difference from peak-to-peak, for all observed modes is $0.45 \pm 0.05 \mu \mathrm{Hz}$, while in the case of the even and odd degree modes are $0.48 \pm 0.05$ and $0.55 \pm 0.07 \mu \mathrm{Hz}$ respectively. Although the difference in amplitude between even and odd degree modes seems to remain, the frequency shift for the even modes is larger than that obtained by Régulo et al. (1994).

Since the time-series created are one year long, the time variation of the integrated frequency shift analyzed here informs only on long-term changes. A recent analysis covering different time scales from one to seven months using 9 years of BiSON data can be found in 
Chaplin et al. (2001). Average values of the following solar activity indices have been computed over the same one year periods than the frequency shifts in order to obtain the corresponding correlation coefficients:

- the International Sunspot Number, $R_{\mathrm{I}}$;

- the integrated radio flux at $10.7 \mathrm{~cm}, F_{10}$;

(both obtained from the Solar Geophysical Data) ${ }^{1}$;

- the Kitt Peak magnetic index (KPMI) extracted from the Kitt Peak full disk magnetograms (Harvey 1984);

- the Mount Wilson Magnetic Plage Strength Index (Ulrich 1991), MPSI;

- the Total Solar Irradiance, TSI (Fröhlich \& Lean 1998) and;

- the equivalent width of HeI $10830 \AA$ averaged over the whole solar disk using data from Kitt Peak observatory.

The Pearson correlation coefficient $r_{\mathrm{P}}$, which is a measure of the strength of the linear relationship between two indices, and the Spearman rank correlation coefficient, $r_{\mathrm{S}}$ which provides a measure of the correlation between the ranks of two indices during the chosen period, are shown in Table 1 for the frequency shift. The correlation between the different solar activity indices themselves have been investigated in details by Bachmann \& White (1994).

In addition, the probability $P_{\mathrm{s}}$ of having null correlation between the ranks of any of the solar indices and the frequency shift is indicated. We note that the database of MPSI and TSI indices, covers only the first 28 time-series. The correlation analysis for these two indices was therefore made with two fewer points than for the others.

The integrated signal shows very high correlation with the various solar indices, whereas the frequency shift corresponding to $\ell=1,3$ and, more significantly, $\ell=0,2$ are slightly lower. The general trend of the frequency shift corresponding to the even and odd degree modes separately is quite clear but, in addition to the difference in amplitude already mentioned, the frequency shifts measured for the even modes seem to be sensitive to the solar cycle later than the odd ones and the resulting difference in phase between the two curves is probably at the origin of the lower correlation coefficients found for $\ell=0,2$. As demonstrated by Moreno-Insertis \& Solanki (2000) different modes would respond differently at different phases of the cycle depending on the positions of the activity (i.e. sunspots) on the disk. Aside of the long term differences, there are also fluctuations at shorter time scales which are different for the two data sets.

Because of the excellent linear correlation coefficients found, the frequency shifts were fitted as a linear function of the different solar indices $I$ by:

$\Delta \nu^{i}=a+b \cdot I^{i}$.

In Table 2 we report the intercepts and the slopes obtained for all the solar indices considered, and an example is given

\footnotetext{
1 Available at http://web.ngdc.noaa.gov/stp/stp.html
}

for the radio flux in the lower part of Fig. 2. The slopes can be compared with the results shown in Table 1 of Régulo et al. (1994) also obtained for low-degree $p$-modes. The three solar indices in common to both works present similar slopes; the differences are less than 3 times our error bars. Notice that, more or less, all activity indices used here produce similar values of the correlation coefficient with the frequency shifts leading to the conclusion than none is much better, for this purpose, than others.

The numbers also agree with those obtained recently by Jain et al. (2000) for intermediate-degrees $(\ell=20-100)$ using GONG data. However, intermediate degree modes are confined closer to the surface and one may expect them to be more sensitive to the activity changes and therefore the slope to be larger for those modes than for low-degree modes. This is indeed what we found analyzing LOWL data (Jiménez-Reyes et al. 2001) but, as we shall see, this depends also strongly on the range of frequencies considered to calculate the average values.

The interpretation of the different behavior found for $\ell=1,3$ and $\ell=0,2$ is not straightforward. In order to understand better the underlying physics, one may instead look at the velocity power variations and at the frequency and $\ell$-dependences. This is considered in the following section.

\section{Total velocity power variations}

The first observations of the variation of the TVP for all measured $p$-modes was reported by Pallé et al. (1990a,b) who found an increase of 30 to $40 \%$, anti-correlated with the solar activity cycle. Afterwards, Anguera Gubau et al. (1992) found similar changes using different analysis techniques and more data of the same type; they interpreted these results as a decrease in the efficiency of the excitation of such modes at solar activity maximum, since absorption of mode power by local magnetic structures (see e.g. Bogdan et al. 1993) is a small influence and cannot explain such a high ratio.

We have also calculated the TVP in the spectrum which is proportional to the area under the main peak of the cross-correlation function. Once the main peak is fitted to a Lorentzian profile, the TVP is calculated as the amplitude times the width (i.e. $T V P^{i}=A^{i} \cdot \Gamma^{i}$ ). We show in Fig. 3, the percentile changes of the TVP compared to its minimum value. Again, the radio flux is shown here as an index of the solar activity cycle. From the figure, we are able to see that the variation of the TVP between minimum and maximum of the solar cycle is around $20 \%$. Moreover, the lower figure shows clearly the anticorrelation with the solar cycle, the TVP decreases when activity increases. The separated contributions of the odd and even pairs of modes were also studied (see the inset box in Fig. 3) as we did in the analysis of the frequency shifts. The results are shown in the sub panel in Fig. 3. Notice that the variation of the TVP between extreme phases of the cycle for even and odd degrees is approximately $20 \%$, in good agreement with the amplitude of the 

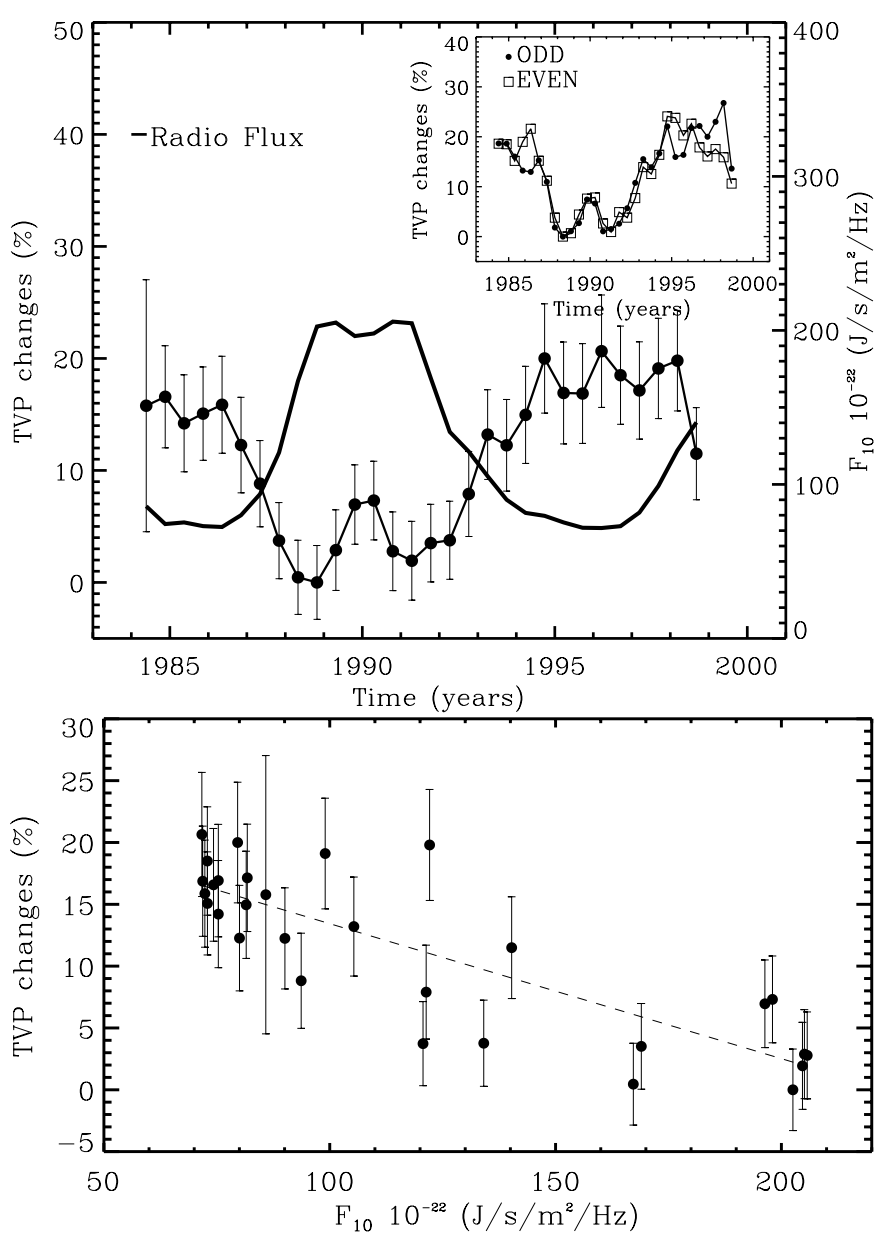

Fig. 3. Time variation of the $T V P$ changes in percent compare to its minimum value (i.e. $\left.100 \cdot\left(T V P^{i}-T V P_{\min }\right) / T V P_{\min }\right)$ for low-degree $p$-modes integrated between 2.5 and $3.7 \mathrm{mHz}$. In the sub-panel the TVP corresponding to $\ell=1,3$ and $\ell=0$, 2 are also shown. The radio flux at $10.7 \mathrm{~cm}$ calculated for the same periods is plotted as a full line in the upper plot and its linear correlation with the frequency shift is shown in the lower plot.

integrated measurement. Moreover, both contributions are in phase, which contrasts with the results found for the frequency shifts, where even modes seems to respond later than the odd ones. Odd and even TVP appear also better correlated around the maximum than during the low activity phases: both present exactly the same bump in the middle of the maximum of activity close to 1990, anticorrelated with decreasing activity during the same period, but their short term variations are different around 1986 and 1996 during the activity minima

Linear and rank correlation coefficients were calculated with the same activity indicators than before and they are shown in Table 3 . The values found are large, showing a good correlation, but they are systematically lower than those for the frequency shift (range [0.67-0.84] against [0.86-0.94]). Moreover, the correlations are bigger for $\ell=0,2$ than for $\ell=1,3$ contrary to the results found for the frequency shifts. The linear correlation coefficient between frequency shift and TVP change $\left(r_{\mathrm{P}}=-0.75\right.$,

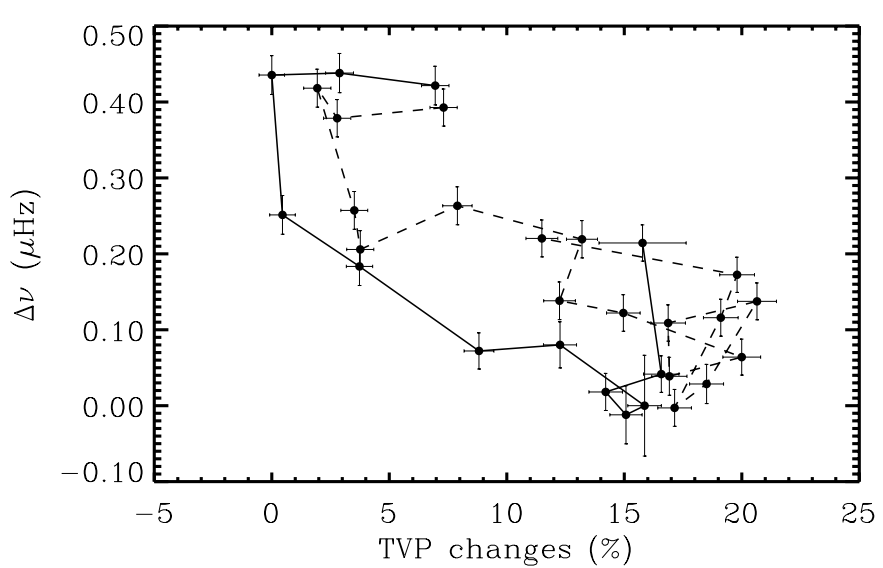

Fig. 4. TVP increase in percent against the frequency shift. The solid line goes from 1984 to 1989 and the dashed line goes from 1990 to 1999 . The corresponding correlation coefficients are shown in Table 3.

see also Table 3 ) is well below the 0.9 reached in average between frequency shift and the solar activity indices suggesting that they are not linearly correlated and Fig. 4 shows indeed that they tend to follow an hysteresis shape, rather than a strict line, when plotted against each other.

This is important because if the decrease in TVP is due to the presence of local or surface activity, as we believe is the cause for the frequency shift, then they should be well correlated with almost no hysteresis. Therefore, the fact that the TVP is less well correlated with the surface activity indices and shows an hysteresis behavior when plotted against the frequency shift, may indicate that indeed its variation is due to a decrease in the excitation efficiency or an increase of the damping rate at maximum which reflects a change in the convection zone structure that does not have to be correlated or strictly in phase with the surface magnetic features. The process of absorption and damping of $p$-modes by an increasing number of rising flux tubes during the period of high activity explored by Bogdan et al. (1996) is qualitatively compatible with our results. On the other hand, if the TVP variations are only due to geometrical effects, this parameter would probably show a better correlation with the total area covered by the magnetic structures than with just the number of them. Although it is beyond the scope of this work, this should probably be further investigated to get a better picture of the possible sources of this phenomena. A more quantitative work including separate observations of the damping rate and amplitude variations of individual modes (Chaplin et al. 2000) is certainly needed to be more conclusive. These inferences from individual mode fits are potentially more informative but remains however less robust than those obtained from the fit of the crosscorrelation of the power spectra.

\section{Frequency dependence of the frequency shifts}

In the previous sections, the variations of the frequencyintegrated velocity power and frequency shifts have been 
Table 3. Correlation coefficients between the TVP and the solar indices where the TVP is defined as the product between the width and amplitude of the cross-correlation function between a spectrum and the 1986 reference spectrum. TVP refers to the average over the whole observed $\ell$ range $(\ell=0,1,2,3)$ whereas $T V P_{0,2}$ and $T V P_{1,3}$ refer to the average for even and odd degrees respectively, all quantities being frequency integrated values between 2.5 and $3.7 \mathrm{mHz}$. The last line gives the correlation coefficients between the TVP and frequency shift. $r_{\mathrm{P}}$ is the Pearson linear correlation coefficient, $r_{\mathrm{S}}$ the Spearman rank correlation coefficient and $P_{\mathrm{s}}$ is the probability of having no correlation.

\begin{tabular}{|c|c|c|c|c|c|c|c|c|c|}
\hline \multirow[t]{2}{*}{ Index } & \multicolumn{3}{|c|}{$T V P$} & \multicolumn{3}{|c|}{$T V P_{0,2}$} & \multicolumn{3}{|c|}{$T V P_{1,3}$} \\
\hline & $r_{\mathrm{P}}$ & $r_{\mathrm{S}}$ & $P_{\mathrm{s}}$ & $r_{\mathrm{P}}$ & $r_{\mathrm{S}}$ & $P_{\mathrm{s}}$ & $r_{\mathrm{P}}$ & $r_{\mathrm{S}}$ & $P_{\mathrm{s}}$ \\
\hline$R_{\mathrm{I}}$ & -0.84 & -0.82 & $4 \times 10^{-8}$ & -0.87 & -0.88 & $1 \times 10^{-10}$ & -0.79 & -0.71 & $1 \times 10^{-5}$ \\
\hline$F_{10}$ & -0.83 & -0.79 & $2 \times 10^{-8}$ & -0.86 & -0.87 & $5 \times 10^{-10}$ & -0.78 & -0.69 & $3 \times 10^{-5}$ \\
\hline KPMI & -0.78 & -0.75 & $2 \times 10^{-6}$ & -0.81 & -0.81 & $7 \times 10^{-8}$ & -0.74 & -0.67 & $6 \times 10^{-5}$ \\
\hline MPSI & -0.82 & -0.80 & $3 \times 10^{-7}$ & -0.83 & -0.85 & $9 \times 10^{-9}$ & -0.80 & -0.69 & $5 \times 10^{-5}$ \\
\hline TSI & -0.76 & -0.67 & $1 \times 10^{-4}$ & -0.80 & -0.76 & $2 \times 10^{-6}$ & -0.70 & -0.58 & $1 \times 10^{-3}$ \\
\hline $\mathrm{He}$ & -0.84 & -0.82 & $2 \times 10^{-8}$ & -0.87 & -0.87 & $4 \times 10^{-10}$ & -0.77 & -0.72 & $8 \times 10^{-6}$ \\
\hline$\Delta \nu$ & -0.75 & -0.73 & $4 \times 10^{-6}$ & & & & & & \\
\hline
\end{tabular}

studied. However, the time variation of the frequency shift is expected to be different at different frequencies. Previous works (e.g. Libbrecht \& Woodard 1990; Anguera Gubau et al. 1992; Chaplin et al. 1998) have shown that the modes at high frequency become more sensitive to the solar cycle. The quality of our time-series and a new method to fit all the spectra at once motivates us to determine the frequency-dependence of the frequency shift for low degrees.

The first method used is related again to the fact that pairs of low-degree acoustic modes are equally spaced in the spectrum. Each spectra is divided in regions of $135 \mu \mathrm{Hz}$ containing a set of modes $\ell=0,1,2$ and 3 . Then, every region is cross-correlated with the corresponding region of the reference spectrum (corresponding to the solar activity minimum of 1986), and the method explained above (Sect. 3) is used to calculate the frequency shift. Finally, the frequency shift of each region is fitted as a linear function of the integrated radio flux at $10.7 \mathrm{~cm}$.

In the second method proposed here, we try to fit together all the spectra at once. We have established that the central frequencies of the solar acoustic modes vary during the solar cycle and there is a strong linear correlation with any of the solar indices. So, in order to improve the statistics we can fit all the spectra together introducing the frequency shift as a new parameter. Here, as well as in the first method, the radio flux was chosen because, according to Table 1, it leads to the best linear correlation coefficients for the central frequency variations but, as quoted before, this choice is not crucial as all indices present similar correlations. We emphasize that, while the first method is faster, the second provides us not only individual frequency shifts but also valuable mode parameters, i.e. mode resonant frequencies corrected for the solar-cycle effects.

As the structure of the power spectrum is complicated by the presence of the $11.57 \mu \mathrm{Hz}$ sidebands, modes close in frequency must be fitted simultaneously in order to maintain the stability in the fits. Therefore adjacent $(n, \ell)$ and $(n-1, \ell+2)$ peaks are fitted together. The multiplet structure induced by the rotation and the temporal sidebands for each mode of the pair are also included in the model. If we label by $p$ the $60 \mu \mathrm{Hz}$ wide part of the spectrum including the adjacent $(n, \ell)$ and $(n-1, \ell+2)$ peaks and $\nu_{p}$ the mean frequency of the pair, the model for this part of the spectrum of each time-series $i$ can be expressed as:

$$
\begin{gathered}
M_{p}^{i}(\nu, \boldsymbol{a})=\sum_{k=-1}^{1} \beta_{|k|}\left[\sum_{m=-\ell}^{\ell} \frac{\alpha_{|m|}^{\ell} A_{n \ell}\left(\Gamma_{p} / 2\right)^{2}}{\left(\nu-\nu_{n \ell m}^{i k}\right)^{2}+\left(\Gamma_{p} / 2\right)^{2}}\right. \\
\left.+\sum_{m=-\ell-2}^{\ell+2} \frac{\alpha_{|m|}^{\ell+2} A_{(n-1)(\ell+2)}\left(\Gamma_{p} / 2\right)^{2}}{\left(\nu-\nu_{(n-1)(\ell+2) m}^{i k}\right)^{2}+\left(\Gamma_{p} / 2\right)^{2}}\right]+B_{p}
\end{gathered}
$$

with:

$\nu_{n \ell m}^{i k}=\nu_{n \ell}+\delta \nu\left(\nu_{p}\right)_{\ell, \ell+2} \cdot\left(F_{10}^{i}-F_{10}^{\mathrm{o}}\right)+m \cdot s_{n \ell}+k_{D} \cdot k,(6)$

where:

$-\nu_{n \ell}$, is the central resonance frequency of the mode $(n, l)$ at solar minimum;

$-\delta \nu\left(\nu_{p}\right)_{\ell, \ell+2}$, is the searched frequency shift per solar index unit, assumed to be the same for all the components of the pair;

$-\nu_{p}=\left(\nu_{n \ell}+\nu_{n-1, \ell+2}\right) / 2$ is the center of the $60 \mu \mathrm{Hz}$ wide interval of the spectrum fitted;

$-s_{n \ell}$, is the synodic rotational splitting for a multiplet taken to be constant for all of them and equal to $400 \mathrm{nHz}$;

- $F_{10}^{i}$, is the averaged radio flux for the given series $\left(F_{10}^{\mathrm{o}}\right.$ being the value at the solar cycle minimum);

- $B_{p}$, is a constant background level at the fitted frequency interval;

- $A_{n \ell}$, is the power at the resonance for sectoral components $m=\ell$

- $\alpha_{|m|}^{\ell}$, is a given ratio $A_{n \ell m} / A_{n \ell}$ constrained to take the theoretical value, for an instrument without spatial resolution, calculated by Christensen-Dalsgaard (1989) for the special case of resonant scattering observation using potassium line; 
$-\Gamma_{p}$, is the linewidth of the components of the multiplet assumed to be the same for all components of the two multiplets; and

$-\beta_{|k|}$, is the ratio of the power of the sidebands $(|k|=1)$ to the central peak $(|k|=0)$. These are directly related to the duty cycle and can be estimated empirically. The duty cycles of the yearly time-series remaining close to their average value of $25 \%$, this allows us to fix the amplitude of the sidebands to $\beta_{|1|}=0.5$ for $\beta_{0}=1$.

We assume that each $m$-component is well represented by a symmetric Lorentzian profile (even though Thiery et al. 2000 have found a slight asymmetry on them) and that the individual $m$-components are independent (see Foglizzo et al. 1998). $B_{p}, A_{n \ell}, \Gamma_{p}, \nu_{n \ell}$ and $\delta \nu_{n \ell}$ are the parameters to be fitted (vector $\boldsymbol{a}$, hereafter). Notice that, in Sect. 4, it has been demonstrated that the TVP depends on the solar activity. This indicates that time variations may also occur for amplitudes and linewidths. Such variations have indeed been found recently from BiSON (Chaplin et al. 2000) for low degree and GONG (Komm et al. 2000) for higher degrees, both showing an increase of the linewidths and a decrease of the amplitudes leading to decrease of the TVP in agreement with our result. We therefore tried to parameterize amplitudes and linewidths as a function of time in the way used for frequencies, but the fits turned out to be very difficult and in many cases did not converge.

At higher frequencies $(\nu>3500 \mu \mathrm{Hz})$ the peaks get wider, the width being greater than their rotational splittings $\left(\Gamma_{p} \gg s_{n \ell}\right)$. Moreover, the linewidths get so large that they become comparable to, or bigger than, the small frequency separations between the pair $(\ell, n),(\ell+2, n-1)$. Therefore the fit is made at frequency intervals (labeled by $q$ hereafter) of $165 \mu \mathrm{Hz}$ centered at $\nu_{q}$ and containing one pair of even modes $(n, 0)(n-1,2)$ labeled hereafter by $p=1$ and one pair of odd modes $(n, 1)(n-1,3)$ labeled hereafter by $p=2$. Only one Lorentzian is fitted for even and another one for odd modes. Thus the model becomes:

$M_{q}^{i}(\nu, \boldsymbol{a})=\sum_{p=1}^{2} \frac{A_{p q}\left(\Gamma_{p q} / 2\right)^{2}}{\left(\nu-\nu_{p q}^{i}\right)^{2}+\left(\Gamma_{p q} / 2\right)^{2}}+B_{q}$,

with:

$\nu_{p q}^{i}=\nu_{p q}+\delta \nu\left(\nu_{q}\right) \cdot\left(F_{10}^{i}-F_{10}^{o}\right)$.

Here the parameters $\boldsymbol{a}$ to be fitted are: $B_{q}, A_{p q}, \Gamma_{p q}, \nu_{p q}$ and $\delta \nu\left(\nu_{q}\right)$.

As Woodard (1984) points out, in the case of observations without spatial resolution, the power spectrum of the solar $p$-mode oscillations is distributed around the mean Lorentzian profiles with a $\chi^{2}$ probability distribution with two degrees of freedom. Consequently, the power spectrum appears as an erratic function where an abundance of frequency fine structure can be found. For this type of statistics the joint probability function associated to the observed power spectrum $\boldsymbol{X}^{i}=\left\{X^{i}\left(\nu_{j}\right)\right\}_{j=1, N}$ corresponding to the time-series $i$ is given by:

$f^{i}\left(\boldsymbol{X}^{i}\right)=\prod_{j=1}^{N} \frac{1}{M^{i}\left(\nu_{j}, \boldsymbol{a}\right)} \exp \left[-\frac{X^{i}\left(\nu_{j}\right)}{M^{i}\left(\nu_{j}, \boldsymbol{a}\right)}\right]$,
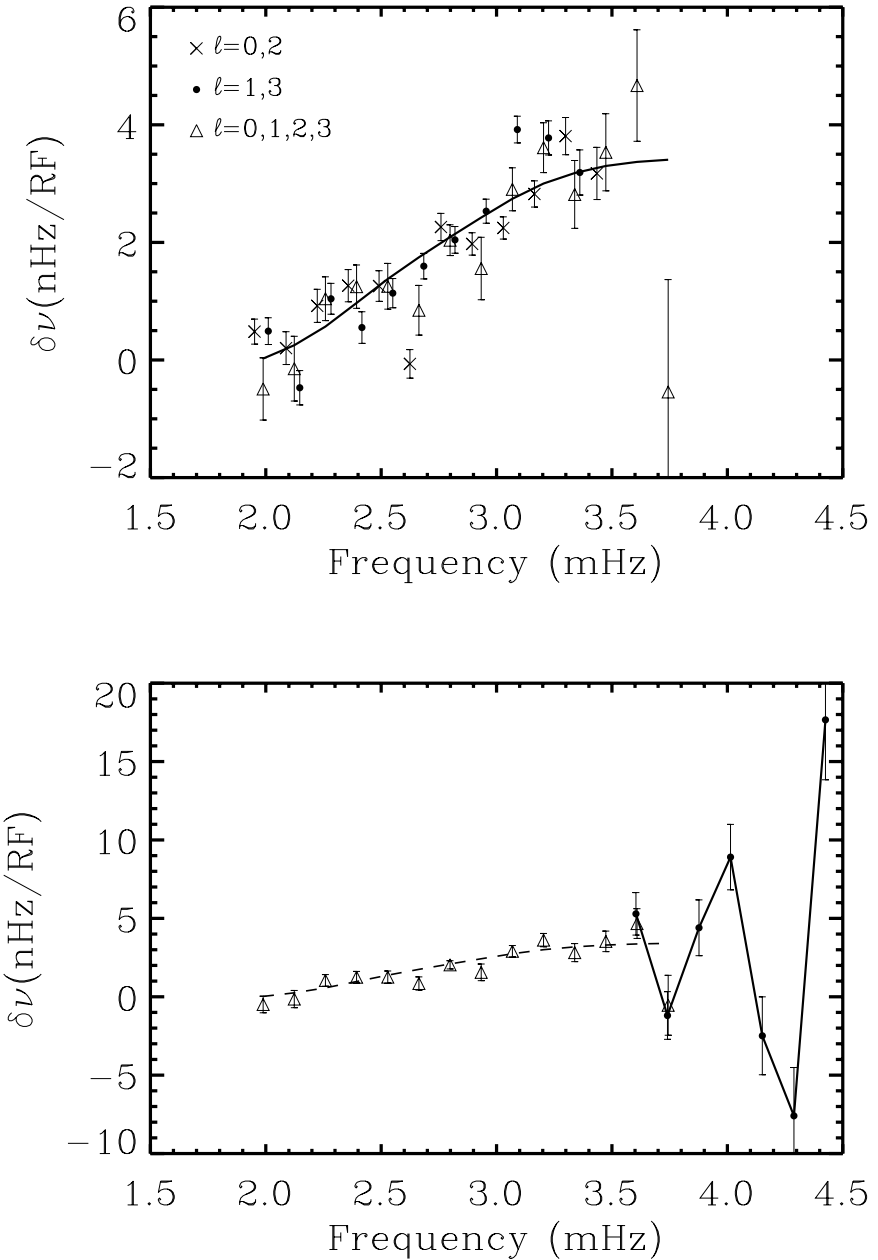

Fig. 5. The slope of the frequency shift in its assumed linear dependence with the radio flux is plotted here as a function of the frequency for low-degree $p$-modes. The results for all modes (triangles) are obtained using the first method described in the text while the separate fits for odd and even degrees are obtained using a simultaneous fit (Eqs. (5), (6)). The solid line is the best fit of the inverse mode mass calculated for the same set of modes. The bottom figure shows the results obtained at high frequency using Eq. (7). The estimation of the frequency shifts corresponding to low degree using the first method as well as the best fit of the inverse mode mass have been again plotted in the bottom figure as reference. RF stands for radio flux units, namely $10^{-22} \mathrm{~J} / \mathrm{s} / \mathrm{m}^{2} / \mathrm{Hz}$.

where $N$ is the number of frequency bins in the interval considered (i.e. the $60 \mu \mathrm{Hz}$ wide interval for $\nu<3.5 \mathrm{mHz}$ or the $165 \mu \mathrm{Hz}$ wide interval for higher frequencies), and $M^{i}$ is given either by Eq. (5) or Eq. (7) depending also on the frequency domain.

The likelihood for the 30 observed spectra is then given by the product of the individual likelihood function for each year. Thus, it can be written as:

$L(\boldsymbol{a})=\prod_{i=1}^{30} f^{i}\left(\boldsymbol{X}^{i}\right)$. 
We then look for the vector $\boldsymbol{a}$ that will maximize the likelihood of the observed spectra according to our model. For numerical reasons one minimizes $S$, defined as the negative logarithm of the likelihood function,

$S(\boldsymbol{a})=\sum_{i=1}^{30} \sum_{j=1}^{N}\left[\ln \left(M^{i}\left(\nu_{j}, \boldsymbol{a}\right)\right)+\frac{X^{i}\left(\nu_{j}\right)}{M^{i}\left(\nu_{j}, \boldsymbol{a}\right)}\right]$

To minimize this expression, we have used a modified Newton method (Press et al. 1992). The initial guesses for the parameters are important to avoid local minima. In that aspect, the frequency shift appears to be the more sensitive parameter and the initial guess was taken from the results shown Table 2 independently of the frequency range fitted. In the case of some parameters (amplitude, noise and linewidth), the natural logarithm of those have been fitted and not the parameters themselves. Doing this, $S$ follows a normal distribution near the minimum and the covariance matrix for the vector $\boldsymbol{a}$ can be approximated by the inverse of the Hessian matrix found at the minimum of $S$. The uncertainties on each fitted parameter are therefore taken as the square roots of the diagonal elements of the inverted Hessian matrix.

The results are summarized in Fig. 5. In the top figure, the triangles represent the frequency shift integrated in bands of $135 \mu \mathrm{Hz}$ using the first method. The frequency dependence is quite clear, the frequency shift being close to zero at $2 \mathrm{mHz}$ and then increasing progressively with the frequency to reach approximately $2 \mathrm{nHz} / \mathrm{RF}$ (where $\mathrm{RF}$ stands for radio flux units, namely $10^{-22} \mathrm{~J} / \mathrm{s} / \mathrm{m}^{2} / \mathrm{Hz}$ ) at the center of the $p$-mode and a maximum of $4 \mathrm{nHz} / \mathrm{RF}$ around $3.6 \mathrm{mHz}$, where it drops fast. The solid line denotes the inverse mode mass extracted from the solar model of Morel et al. (1997). It has been averaged over the same regions in frequency and what we show represent the best fit to the data. The results corresponding to the simultaneous fits are also shown in the same figure. The frequency shift for the even modes are represented by crosses while black circles represent the odd ones. Both confirm the previous results showing a similar frequency dependence. These results are also in agreement with the analysis carried out by Chaplin et al. (1998) on BiSON data for frequency below $3.6 \mathrm{mHz}$. The mean $\langle\delta \nu(\nu)\rangle$ in the $2.5-3.7 \mathrm{mHz}$ range can be estimated by integrating the best fit of the inverse mode mass to our results divided by the length of the frequency range i.e. $1.2 \mathrm{mHz}$. This leads to a value of $2.66 \mathrm{nHz} / \mathrm{RF}$ compatible with the slope $b$ reported in Table 2 for the linear dependance between the integrated frequency shift and radio flux. A comparison of Eqs. (1) and (4) gives the straightforward correspondance between $b$ and the averaged frequency shift per radio flux units. We note however that the two estimates are not equivalent due to the variation of the $p$-mode energy across the five minute band. The cross-correlation function weight more the peak with higher amplitude located around $\sim 2.9-3.0 \mathrm{mHz}$ while the present integration gives the same weight to all the modes.
At high frequency, the first points corroborate the frequency shift obtained using the first method, where a sharp downturn is found. Then, at higher frequency, a large fluctuation appears. A similar feature was found by Anguera Gubau et al. (1992) and also found for intermediate degree by Libbrecht \& Woodard (1990) in the analysis of BBSO data. These authors observed that the sensitivity of the mode frequency shifts show similar sharp downturn located around $3.8 \mathrm{mHz}$. Chaplin et al. (1998) also reported (in their Fig. 4) a sharp downturn with negative frequency shift of about $-10 \mathrm{nHz} / \mathrm{RF}$ around $4.3 \mathrm{mHz}$ but they did not find the first downturn found at $3.75 \mathrm{mHz}$ as in our analysis. The better sampling of the Mark-I data set and the fact that this first downturn was obtained with the two different methods we used for low and high frequencies make us confident in this result; moreover, this result is also found by Anguera Gubau et al. (1992).

The frequency dependence of the frequency shift has been addressed from the theoretical point of view in different works. The model developed by Goldreich et al. (1991) suggests that a combination of an increase of the chromospheric temperature and a chromospheric resonance can be responsible for the sharp downturn at high frequency followed by an oscillation while the progressive increase in the five-minute band can be interpreted as an increase of the filling factor of the small scale photospheric magnetic fields. On the other hand, Jain \& Roberts $(1993,1996)$ argue that the presence of a magnetic field in the chromosphere and a combination of temperature and magnetic field strength variations could, qualitatively, explain the observed frequency dependence of the frequency shift at both low and high frequencies. We notice that both models, those of Goldreich et al. (1991) and Jain \& Roberts (1996) present a wavelike behavior at high frequency qualitatively similar to the one found in our analysis. The particular model of Goldreich et al. (1991) is even able to reproduce quantitatively this result, including the upturn around $4.5 \mathrm{mHz}$ but no evidence has been found for the required chromospheric resonance (Woodard \& Libbrecht 1991) and Kuhn (1998) point out that the change in the photospheric magnetic field strength needed in this model is much higher than the one obtained from recent infrared splitting observations of the quiet region field strengths or MDI magnetograms. Thus, Kuhn (1998) argues that photospheric magnetic fluctuations are unlikely to be responsible for the observed frequency shifts and proposes instead that turbulent pressure and mean solar atmosphere stratification variations resulting from entropy perturbations through the solar cycle may be the dominant process affecting $p$-mode frequencies. In this model, the associated temperature fluctuations may originate from near the base of the convection zone but in order to explore those possibilities and locate the different possible perturbations one needs to invert the even splitting coefficients (see Dziembowski et al. 2000) and track any fluctuation in the sound speed inversion using long term observations. We intend to extend our work in that direction using the 


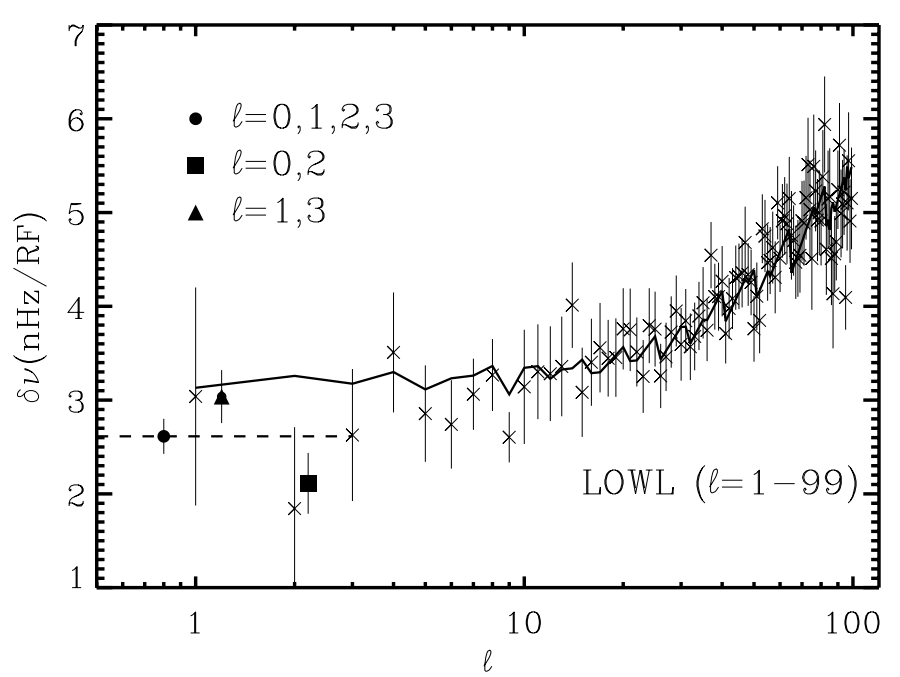

Fig. 6. The cross shows the normalized frequency shift for $\ell=$ 1-99 integrated between 2.5 and $3.7 \mathrm{mHz}$ using LOWL data, and the solid line denotes the best fit of the inverse mode mass for those modes. We have also plotted the results corresponding to very low degree using Mark-I data (Table 2): the dashed horizontal bar is for $\ell=0,1,2,3$; the triangle for $\ell=1,3$ is placed close to $\ell=1$ because this component gives the main contribution; and the square for $\ell=0,2$ has been arbitrarily placed close to $\ell=2$.

observations of both low and intermediate degrees from LOWL and Mark-I.

\section{Analysis of the I-dependence of the frequency shifts}

The LOWL instrument (Tomczyk et al. 1995), located in Mauna Loa, Hawaï, is a Doppler imager based on a Potassium Magneto-Optical Filter, and it has been collecting solar observations for more than six years. With the installation of a similar experiment at the Observatorio del Teide, a new network called ECHO (Experiment for Coordinated Helioseismic Observations) intends to continue the solar observations for a complete solar cycle (Tomczyk et al. 2000) with an increased duty cycle. Recently, six years of data have been re-analyzed through a new pipeline producing mode parameters for low and intermediate degrees (Jiménez-Reyes 2001). We have used the mode frequencies given by this analysis to compare the $\ell$ - and frequency-dependence of the frequency shift at low and intermediate degrees.

Figure 6 shows the normalized frequency shift using LOWL observations for $\ell=1$ up to 99 . It has been performed using those modes between 2.5 and $3.7 \mathrm{mHz}$, as we did for low degree. The inverse mode mass, which was calculated as well for each one of the modes fitted and then averaged in the same way, follows remarkably well the results. The figure shows as well the $\ell$-dependence of low degree $p$-mode frequency shifts for Mark-I found in Sect. 3. The general trend confirms the $\ell$-dependence of the frequency shift, the sensitivity at high degree $(\ell=100)$ being almost twice to that of low-degree. We notice that

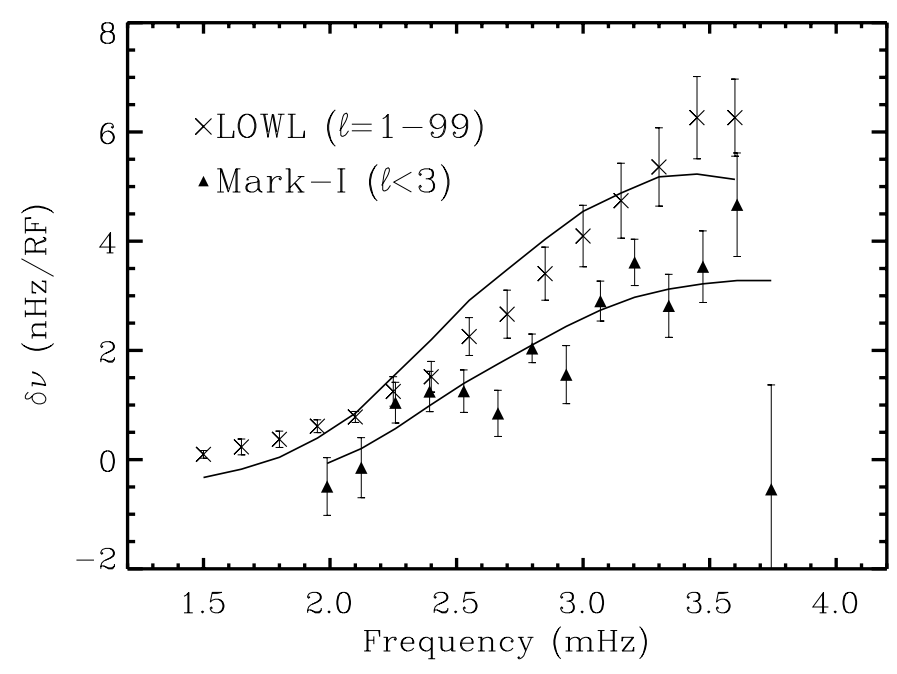

Fig. 7. Normalized frequency dependence of LOWL data averaged for modes from $\ell=1$ up to 99 in intervals of $150 \mu \mathrm{Hz}$. The results for very low degree shown in Fig. 5 are also plotted. The solid lines represent the best fit of the inverse mode mass to both data sets.

the LOWL $\ell=2$ and Mark-I $\ell=0,2$ are in good agreement and significantly lower than the inverse mode mass curve. However the LOWL data error bars in $\delta \nu$ for $\ell=1$ and $\ell=2$ overlap significantly and the small value for $\ell=0,2$ could either be due to particularly small $\delta \nu$ in $\ell=2$ but also in $\ell=0$. It will therefore be important to check this results with independent observations in the future. This is important because if a geometrical effect purely related to the integrated disk measurements affect the even modes measured by Mark-I, this should not be seen for the LOWL resolved measurements. If confirmed, this lower value of the frequency shift for $\ell=2$ may therefore have a physical origin and be the signature of a localized perturbation.

Figure 7 reproduces the frequency dependence for low degree shown in Fig. 5 together with the frequency shift averaged for $\ell$ 's between 1 up to 99 in intervals of $150 \mu \mathrm{Hz}$ obtained from LOWL data. Again, the best fit to the inverse mode mass is shown. The ratio between the two slopes in the inverse mode mass fits is 0.74 and is nearly equal to the ratio between the mean mode mass calculated for both mode sets $(0.75)$ showing that the $\ell$-dependence of the frequency shift is again well described by the $\ell$ dependence of the inverse mode mass. However, although the sensitivity is higher for higher degree modes (LOWL data), the fit is worse than for low degree (Mark-I data); moreover, in this later case, while roughly consistent with the sizes of the error bars, the scatter seems to be organized as an oscillation on top of the fitted line. The higher sensitivity of the frequency shift at high degree seems to be in contradiction with what Régulo et al. (1994) pointed out, but in agreement with recent analysis of Chaplin et al. (1998). While all results point towards the existence of a perturbation confined close to the surface, there is still no convincing evidence of another cause that could exist deeper down as suggested by Régulo et al. (1994). On the 
other hand, the oscillation, also pointed out by Anguera Gubau et al. (1992), that seems to be present in the results for low degree, has the same period $(\approx 400-450 \mu \mathrm{Hz})$ that the one that is clearly found at higher frequencies (see Fig. 5 bottom) and deserves further attention and confirmation.

Finally, we notice that the frequency dependence of the frequency shift obtained from the low degree modes of LOWL data is in very good agreement with the result plotted here using Mark-I data. This, added to the fact that the 6 years of LOWL data were covered also by Mark-I observations, make us very confident in the validity of our analysis of the $\ell$-dependence using both instruments.

\section{Conclusion}

An analysis of the low-degree p-mode frequency shifts over more than a solar cycle has been carried out. Time dependence of the resonant frequencies and the total velocity power have been studied with several, old and new, methods, yielding a correlation and an anti-correlation with the solar activity cycle respectively which confirms previous results. Moreover, quantitative parameters have been introduced which yield the sensitivities of such variations with respect to a given activity index, in our case the radio flux at $10.7 \mathrm{~cm}$. The existence of a hysteresis behavior between both parameters seems to imply different direct causes for them.

Also, the frequency shifts have been obtained as a function of frequency and degree. These studies make use of data taken from two experiments (Mark-I and LOWL) which confirm each other results, when they coincide (at very low $\ell$ modes), and complement their findings otherwise. Indeed, the main source for the variation of the frequencies with the cycle is located near the surface while a secondary, deeper rooted source, seems to be very weak if it exists at all. Interesting details such as the oscillation found for very low- $\ell p$-modes and the surprisingly small frequency shift for $\ell$ even modes need further investigation.

The results obtained and methods used in this work will also be useful in the analysis of very long time-series covering more than half the solar activity cycle, since both resonant frequencies and, to a lesser extent, amplitudes vary cyclically with time, providing wrong results if only data taken at parts of the cycle are used. In the following paper (second part of this work) we will use these methods to minimize the effects of solar activity on these parameters in annual power spectra, allowing an average of all data thus improving the signal to noise ratio. Using this power spectra we will be able to give a new estimation of the solar rotational splitting, which combined with LOWL data, will be used to infer the solar rotation close to the core.

Finally we note that the hysteresis between frequencies of odd and even modes or between mode frequencies and magnetic flux is not addressed in this work based on yearly time series. These studies are however of considerable importance (see e.g. Moreno-Insertis \& Solanki 2000) but would require shorter time series and better data (e.g. higher duty cycle) in order to reliably confirm previous analysis. This should probably be addressed in the future using long term observations of low degree modes from the ground based networks BiSON and IRIS++ (i.e. IRIS + LOWL + Mark-I, Salabert 2001).

Acknowledgements. We are deeply thankful to the all members, past and present, of the helioseismology group at the IAC for doing Mark-I observations and maintenance. The use of Birmingham University resonant scattering spectrophotometer at Observatorio del Teide is also deeply acknowledged. We are extremely grateful to Tom Bogdan and Mausumi Dikpati for useful discussions and additional comments. T. Corbard acknowledges support from NASA grant S-92678-F and PPARC grant PPA/A/S/2000/00171. The High Altitude Observatory of the National Center for Atmospheric Research is sponsored by the National Science Foundation.

\section{References}

Anguera Gubau, M., Pallé, P. L., Peréz Hernández, F., Régulo, C., \& Roca Cortés, T. 1992, A\&A, 255, 363

Bachmann, K. T., \& White, O. R. 1994, Sol. Phys., 150, 347

Bogdan, T. J., Brown, T. M., Lites, B. W., \& Thomas, J. H. 1993, ApJ, 406, 723

Bogdan, T. J., Hindman, B. W., Cally, P. S., \& Charbonneau, P. 1996, ApJ, 465, 406

Chaplin, W. J., Elsworth, Y., Isaak, G. R., Miller, B. A., \& New, R. 2000, MNRAS, 313, 32

Chaplin, W. J., Elsworth, Y., Isaak, G. R., et al. 1998, MNRAS, 300, 1077

Chaplin, W. J., Elsworth, Y., Isaak, G. R., et al. 2001, MNRAS, 322, 22

Christensen-Dalsgaard, J. 1989, MNRAS, 239, 977

Deubner, F., \& Gough, D. 1984, ARA\&A, 22, 593

Dziembowski, W. A., Goode, P. R., Kosovichev, A. G., \& Schou, J. 2000, ApJ, 537, 1026

Foglizzo, T., Garcia, R. A., Boumier, P., et al. 1998, A\&A, 330, 341

Fossat, E., Gelly, B., Grec, G., \& Pomerantz, M. 1987, A\&A, 177, L47

Fröhlich, C., \& Lean, J. 1998, Geophys. Res. Lett., 25, 4377

Goldreich, P., Murray, N., Willette, G., \& Kumar, P. 1991, ApJ, 370, 752

Harvey, J. W. 1984, in CP, vol. 2310, Workshop on Solar Irradiance Variations on Active Region Time Scales, ed. B. La Bonte, G. Chapman, H. Hudson, \& R. C. Wilson (Washington: NASA), 197

Jain, K., Tripathy, S. C., \& Bhatnagar, A. 2000, ApJ, 542, 521

Jain, R., \& Roberts, B. 1993, ApJ, 414, 898

Jain, R., \& Roberts, B. 1996, ApJ, 456, 399

Jiménez-Reyes, S. J. 2001, Ph.D. Thesis, La Laguna University Jiménez-Reyes, S. J., Corbard, T., Pallé, P. L., \& Tomczyk, S. 2001, in SOHO-10 GONG 2000: Helio- and Asteroseismology at the dawn of the millennium (ESA), SP, vol. 464, 107

Jiménez-Reyes, S. J., Régulo, C., Pallé, P. L., \& Roca Cortés, T. 1998, A\&A, 329, 1119

Komm, R. W., Howe, R., \& Hill, F. 2000, ApJ, 531, 1094

Kuhn, J. R. 1998, in Structure and Dynamics of the Interior of the Sun and Sun-like Stars (ESA), SP, vol. 418, 871 
Libbrecht, K. G., \& Woodard, M. F. 1990, Nature, 345, 779

Morel, P., Provost, J., \& Berthomieu, G. 1997, A\&A, 327, 349

Moreno-Insertis, F., \& Solanki, S. K. 2000, MNRAS, 313, 411

Pallé, P. L., Fossat, E., Régulo, C., et al. 1993, A\&A, 280, 324

Pallé, P. L., Pérez, J. C., Régulo, C., Roca Cortés, T., \& Isaak, G. R. 1986, A\&A, 169, 313

Pallé, P. L., Régulo, C., \& Roca Cortes, T. 1989, A\&A, 224, 253

Pallé, P. L., Régulo, C., \& Roca Cortés, T. 1990a, in Progress of Seismology of the Sun and Stars, ed. Y. Osaki, \& H. Shibahashi (Springer-Verlag), LNP, vol. 367, 189

Pallé, P. L., Régulo, C., \& Roca Cortés, T. 1990b, in Progress of Seismology of the Sun and Stars, ed. Y. Osaki, \& H. Shibahashi (Springer-Verlag), LNP, vol. 367, 129

Press, W. H., Teukolsky, S. A., Vetterling, W. T., et al. 1992, Numerical Recipes in Fortran 77: the art of scientific computing (2nd edition) (Cambridge: Cambridge University Press)

Régulo, C., Jiménez, A., Pallé, P. L., Hernández, F. P., \& Cortés, T. R. 1994, ApJ, 434, 384
Salabert, D. 2001, in SOLSPA 2001 Euroconference: Solar Cycle and Space Weather, Vico Equense (Italy), ESA SP477 , in press

Thiery, S., Boumier, P., Gabriel, A. H., et al. 2000, A\&A, 355, 743

Tomczyk, S., Jiménez Reyes, S. J., Jiménez, A., \& Pallé, P. L. 2000, in AAS/Solar Physics Division Meeting, 32, 0117

Tomczyk, S., Streander, K., Card, G., et al. 1995, Sol. Phys., 159,1

Ulrich, R. K. 1991, Adv. Space Res., 11, 217

van der Raay, H. M., Palle, P. L., \& Roca Cortes, T. 1985, in NATA ASI, vol. 169, Seismology of the Sun and the distant stars, ed. D. Gough, 333

Woodard, M. F. 1984, Ph.D. Thesis, University of California, San Diego

Woodard, M. F., \& Libbrecht, K. G. 1991, ApJ, 374, L61

Woodard, M. F., Libbrecht, K. G., Kuhn, J. R., \& Murray, N. 1991, ApJ, 373, L81

Woodard, M. F., \& Noyes, R. W. 1985, Nature, 318, 449 